\title{
On some strange features in Kepler EB light curves
}

\author{
J. Nuspl ${ }^{1}$, T. Hajdu ${ }^{1}$ and T. Hegedüs ${ }^{2}$ \\ 1 Konkoly Observatory, Hungary, (E-mail: nuspl@konkoly.hu) \\ 2 SZTE Baja Astronomical Observatory, Hungary
}

Received: October 31, 2019; Accepted: December 31, 2019

\begin{abstract}
We have scrutinized the approximately 3 thousand compiled eclipsing binary light curves of the Kepler mission in detail and found several typical and repeated common artifacts. We will present some of them and point out their possible origin.
\end{abstract}

Key words: binary stars - data processing

\section{Introduction}

The Kepler Eclipsing Binary Catalog - Third Revision contains data for about 3000 systems (Prša et al, 2011). Enumerate all the papers connected to the database we could get around 1300 papers in the literature published during the last decade, so its impact is definitely huge.

One could suppose that this uniquely large database with unprecedented accuracy would provide a good foundation for detailed studies as it was advocated, e.g. in Wilson (2003), to look for an efficient, effective, and automatic processing of massive database secured by large-scale surveys but 'Eclipsing binary (EB) modeling naturally partitions into several areas, headed by the overall issue of how to find astrophysically useful numbers.' (Wilson, 2006) However, the number of studies discussing astrophysical details is much more limited.

Pursuing this main goal, we have browsed through the catalog of corrected and detrended light curves in the database, and have found a few typical features created during data processing. These detections demonstrate that one must be careful around the details before using the data directly to deduce any physical conclusion. The artificial side effects contaminate the real data and can mimic otherwise pretty intriguing real effects.

\section{Discussion}

The first plot shows an upward trend distortion around eclipses arising from the applied detrending procedure. However, from a purely mathematical viewpoint, it could come from Gibbs overshooting at breakpoints of smooth functions cleaning the data. From physical point of view, considering possible non-radially 


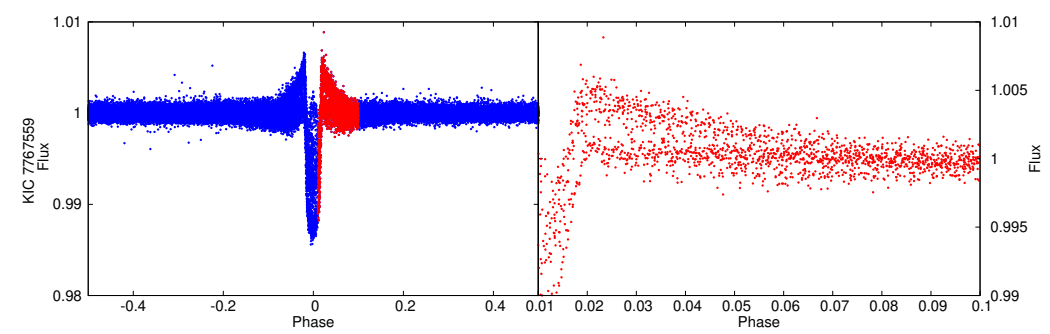

pulsating components in the system, it might be a physical effect from the amplification and modulation of hidden modes $(1+\mathrm{m}=$ odd $)$ during partial eclipses. Even so, zooming into the plot one can disclose these latter origins and conclude that it comes from the data processing.

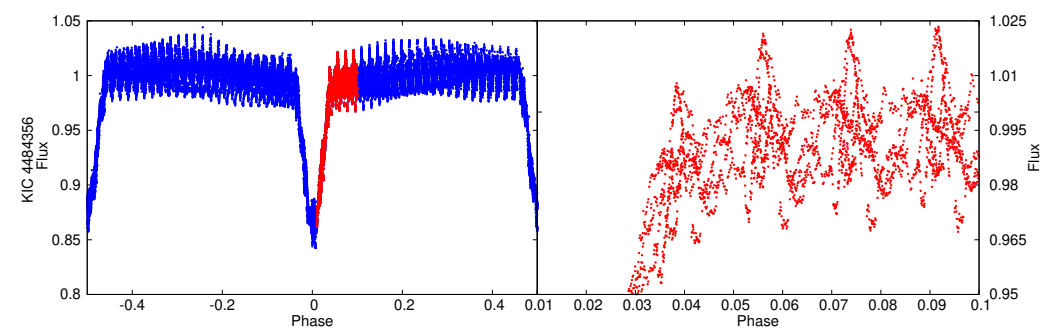

The phase folded light curve shows seemingly a pulsation like pattern. If it came from a physical oscillation, it would only be possible with locked synchronization to the orbital motion. Zooming into the plot the structure reveals that the observed pattern cannot be a real one. Considering the long term behavior one can recognize some slow variability of it, the real origin of the oscillation in the data series. The observed structure is only a moire noise pattern, due to that the ratio between the sampling rate commensurate with the period of variation and the period search algorithm could get better fitting with an incorrect value.

Acknowledgements. This work was supported by OTKA K-129249 and NN129075 and Lendület Program of the HAS, No. LP2018-7/2018.

\section{References}

Prša, A. et al., 2011, $A J, \mathbf{1 5 1}, 101$

Wilson, R.E.,2003, $A S P C, \mathbf{2 9 8}, 313$

Wilson, R.E.,2006, $A S P C, \mathbf{3 4 9}, 71$ 